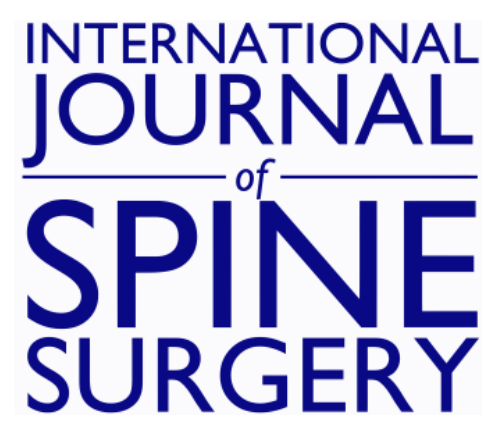

\title{
Image Guidance in Spinal Surgery: A Critical Appraisal and Future Directions
}

Fabian Sommer, Jacob L. Goldberg, Lynn McGrath, Jr, Sertac Kirnaz, Branden Medary and Roger Härtl

Int J Spine Surg 2021, 15 (s2) S74-S86

doi: https://doi.org/10.14444/8142

http://ijssurgery.com/content/15/s2/S74

This information is current as of April 26, 2023.

Email Alerts Receive free email-alerts when new articles cite this article. Sign up at:

http://ijssurgery.com/alerts

The International Journal of Spine Surgery

2397 Waterbury Circle, Suite 1,

Aurora, IL 60504, Phone: +1-630-375-1432 


\title{
Image Guidance in Spinal Surgery: A Critical Appraisal and Future Directions
}

\author{
FABIAN SOMMER, MD, JACOB L. GOLDBERG, MD, LYNN MCGRATH JR, MD, SERTAC KIRNAZ, MD, \\ BRANDEN MEDARY, BS, ROGER HÄRTL, MD \\ Department of Neurological Surgery, Weill Cornell Medicine, New York Presbyterian Hospital, New York, New York
}

\begin{abstract}
Background: Image-guided spinal surgery (IGSS) underwent rapid development over the past decades. The goal of IGSS is to increase patient safety and improve workflow. We present an overview of the history of IGSS, illustrate its current state, and highlight future developments. Currently, IGSS requires an image set, a tracking system, and a calibration method.

Imaging: Two-dimensional images have many disadvantages as a source for navigation. Currently, the most common navigation technique is three-dimensional (3D) navigation based on cross-sectional imaging techniques such as cone-beam computed tomography (CT) or fan-beam CT.

Tracking: Electromagnetic tracking uses an electromagnetic field to localize instruments. Optical tracking using infrared cameras has currently become one of the most common tracking methods in IGSS.

Calibration: The three most common techniques currently used are the point-matching registration technique, the surface-matching registration technique, and the automated registration technique.

Future: Augmented reality (AR) describes a computer-generated image that can be superimposed onto the realworld environment. Marking pathologies and anatomical landmarks are a few examples of many possible future applications. Additionally, AR offers a wide range of possibilities in surgical training. The latest development in IGSS is robotic-assisted surgery (RAS). The presently available data on RAS are very encouraging, but further improvements of these procedures is expected.

Conclusion: IGSS significantly evolved since its inception and is becoming a routinely used technology. In the future, IGSS will combine the advantages of "active/freehand 3D navigation" with AR and RAS and will one day find its way into all aspects of spinal surgery, not only in instrumented procedures.

Special Issue Article

Keywords: image-guided surgery, tracking, calibration, navigation, augmented reality, robotic, spine surgery
\end{abstract}

\section{INTRODUCTION}

Advancements in visual assistance technology have facilitated breakthroughs in surgery, from the invention of loupes to the introduction of the surgical microscope and now image-guided spinal surgery (IGSS). IGSS technologies have been introduced, tested methodically, and integrated into operating rooms (ORs). ${ }^{1}$ The primary goal of this new technology is to increase safety for the patient. In addition, in recent years, the ability of technology to reduce the psychological and physical effort required from the surgeon is recognized as having a beneficial effect on outcomes. ${ }^{2,3}$ Especially the latter point explains the expansion of IGSS not only to complex procedures but also to spinal procedures that are considered more routine and the stream- lining of the surgical workflow is being considered of value by the surgeon and the OR staff.

The rapid and exponential increase in computing power over the past several decades has supported applications such as IGSS. ${ }^{4}$ At the same time, the surgical technology development industry has also discovered this field as a new market and has developed together with surgeons a variety of different assistance systems. ${ }^{5-7}$ The common goal across all support systems is that the surgeon's orientation should be facilitated, and the accuracy of the intervention should be improved. For this reason, anatomically difficult surgical procedures, such as those with proximity to critical anatomical structures, nerves, and vasculature, are especially of interest for intraoperative navigation. Similarly, the use of navigation seems to be particularly beneficial for procedures involving difficult visualization. ${ }^{6,7}$ 
Minimally invasive spine surgery frequently involves operating in these difficult conditions. For example, these techniques require surgical instruments to be passed through smaller incisions and in close anatomical proximity to critical structures, such as the spinal cord, aorta, vena cava, and/or vertebral arteries. $^{8}$

The proficient use of navigation has become essential to the successful performance of a wide range of minimally invasive procedures. ${ }^{9}$ Its utility has improved tremendously since its early iterations. Widely available navigation systems allow for the real-time three-dimensional (3D) visualization and localization of surgical instruments in relation to the patient's intraoperative computed tomography (CT)-based anatomy. ${ }^{6,10}$ As navigation systems become "main stream" with more routine use in spine surgery, we present here a brief overview of the history and development of intraoperative spine navigation. Additionally, we illustrate the current state of navigation technology in spine surgery. Lastly, we highlight several exciting future developments and our expectations for the future of spinal navigation.

\section{EVOLUTION OF COMPUTER-AIDED SURGERY}

In 1971, the first published reports suggesting that the use of computers could aid surgeons occurred. ${ }^{11}$ The procedures described therein were far from navigated surgery as we know it today. In 1973, the first neurosurgical description of a procedure for computer-assisted determination of intracranial coordinates during stereotactic procedures using a Leksell frame was published. ${ }^{1}$ This was the beginning of the actual development of computer-assisted surgery (CAS). The fact that the origins of navigation took place in stereotactic cranial surgery was no surprise; in this field, it is of paramount importance to find the smallest possible surgical approach to the pathology and to correct the pathology as precisely as possible without affecting surrounding tissue. ${ }^{12}$ The spine is very comparable in this aspect, but it is anatomically more difficult to approach for navigation. ${ }^{13}$ As a result, it took more than 20 years after the initial description of a computer-assisted stereotactic navigation procedure until its application in spinal surgery, which was described in 1995. The first publication on the use of a navigation-based procedure in spine surgery was a study by Lavalle

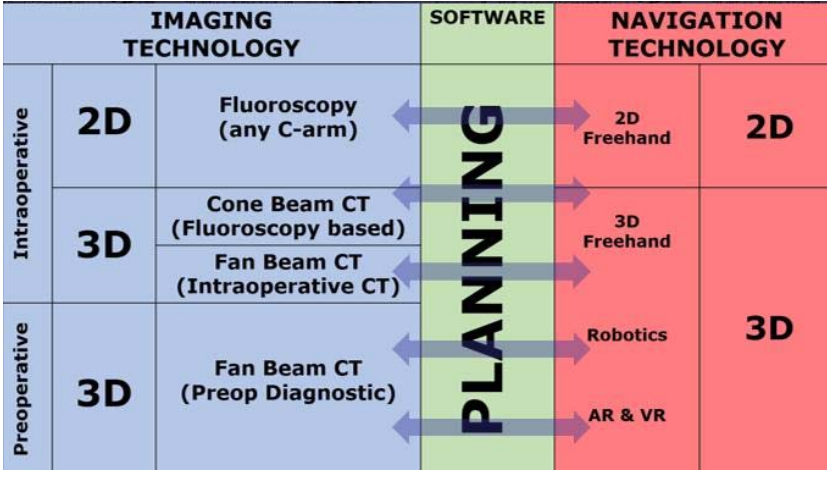

Figure 1. Overview and evolution of image-guided spinal surgery (IGSS) Imaging and navigation technologies are connected by planning software. Imaging and navigation technology evolve in tandem. Cone beam computed tomography (CT) scanners are C-arm-based imaging platforms that obtain multiple images through a "cone-shaped" x-ray beam during a $190^{\circ}$ rotation around the patient. Examples are the O-arm (Medtronic, Dublin, Ireland) or the Ziehm RFD3D (Ziehm Imaging $\mathrm{GmbH}$, Nuremberg, Germany). Intraoperative fan beam CT scanners such as the AIRO (Brainlab AG, Munich, Germany) are portable CT platforms that emit a fan-like $\mathrm{x}$-ray that is detected by a linear detector array. Robotic spinal navigation requires preoperative planning of screw positions, which limits its utility to the portion of the case in which screws are placed. By contrast, active navigation allows the surgeon to incorporate the benefits of navigation in each stage of the case from incision planning to bony resection to hardware implantation. Augmented reality $(A R)$ and virtual reality (VR) expand the role for IGSS into more complex surgical procedures but also into the fields of surgical training and patient education.

et al who described the placement of pedicle screws using a CT dataset and an optical 3D localizer. ${ }^{14}$ Over time, various techniques for navigation were developed. ${ }^{6}$ Each navigation procedure currently requires an image dataset by which the navigation software is oriented, a tracking system by which the navigation module determines the position of the instruments, and a calibration so that the image dataset and the position of the surgical instruments are matched (Figure 1). ${ }^{15}$ Over time, different procedures have been developed for all three areas.

\section{IMAGING IN NAVIGATION}

Due to its wide availability and relatively simple processing of the image data, fluoroscopy represented a relatively early used method in CAS or IGSS. ${ }^{16}$ In the past, CT scanners were comparatively large, and the processing speed of the computers was not yet high enough to guarantee fast processing of the datasets for intraoperative navigation. ${ }^{17}$ Foley et al described a method for navigated probing of the pedicles of the lumbar spine relatively early. In their study, they described a method on a cadaver with which a tracked probe could be projected in real time superimposed onto previously acquired x-ray images of the lumbar spine, and thus the virtual trajectory could be 

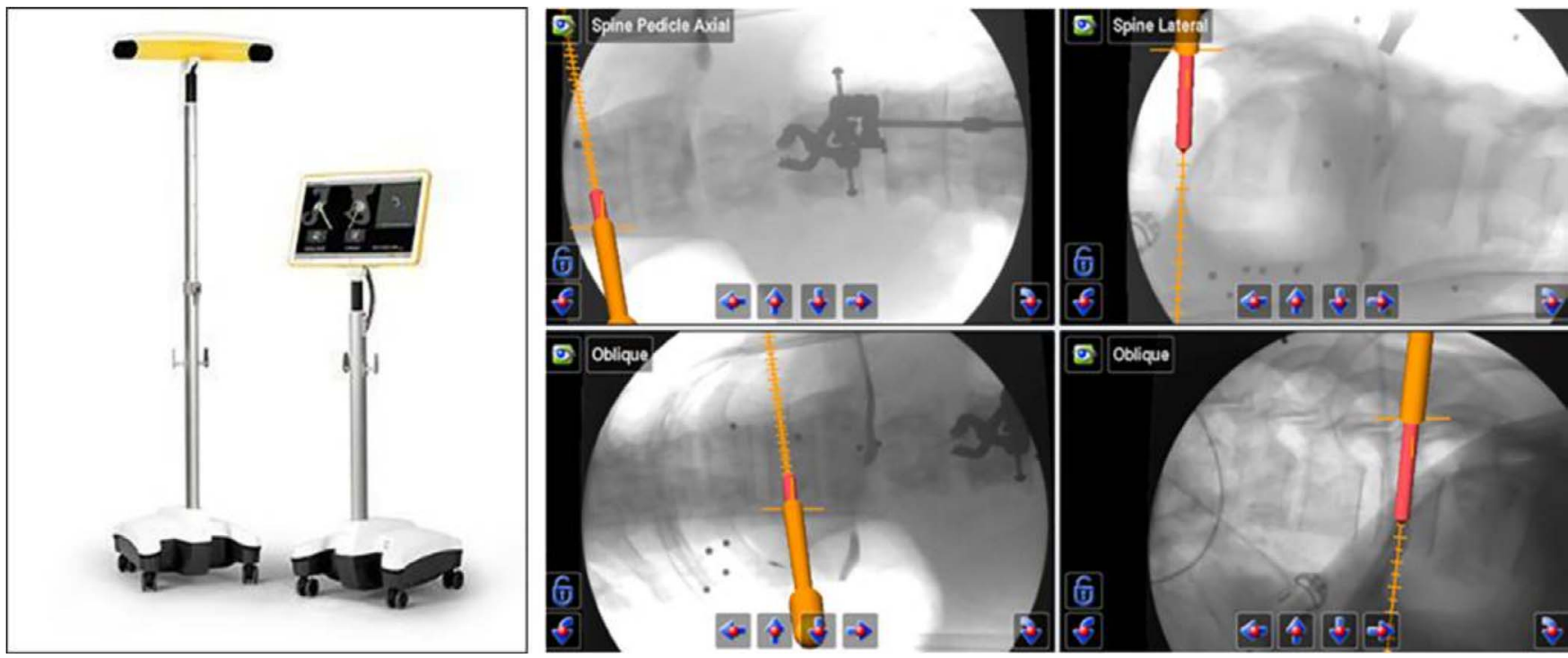

Figure 2. Fluoroscopic-based two-dimensional navigation for pedicle screw placement (Kick system, Brainlab).

appreciated. The tracking of the probe was performed by an optical method using light-emitting diodes. With this method, a relatively high accuracy could already be achieved. ${ }^{16}$ Nevertheless, twodimensional (2D) images have many disadvantages as a source for navigation. For example, it is not possible to display the images without superimposing them into the field of view on top of the surgical site without good depth perception due to the lack of simulated 3D volumes, which is a particular problem in the area of the lower cervical and thoracic spine or in spinal diseases such as ankylosing spondylitis. ${ }^{18}$ This is a striking disadvantage, particularly in comparison to CT-based 3D navigation procedures. However, the radiation exposure during fluoroscopy is also lower. ${ }^{19}$

As computing power and portability of CT scanners increased their intraoperative utility, intra- operative CT with 3D navigation has largely supplanted 2D fluoroscopy-based CAS. ${ }^{20-22}$ Nevertheless, there are still navigation systems based on 2D x-ray images available in routine applications today, which have been able to demonstrate a high level of usability and good surgical results (Figure 2). ${ }^{5}$

Currently, the most common navigation technique is $3 \mathrm{D}$ navigation based on cross-sectional imaging techniques such as cone-beam CT (CBCT) (eg, the Ziehm RFD 3D [Ziehm Imaging $\mathrm{GmbH}$, Nuremberg, Germany]) or fan-beam CT (FBCT) (eg, the Brainlab Airo [Brainlab AG, Munich, Germany]). ${ }^{9,20-22}$ The O-arm (Medtronic, Dublin, Ireland) represents a subset of CBCTs and is therefore not discussed separately (Figure 3). ${ }^{21,23,24}$ Both modalities are very convenient to use, and modern CBCT systems are not noticeably larger or
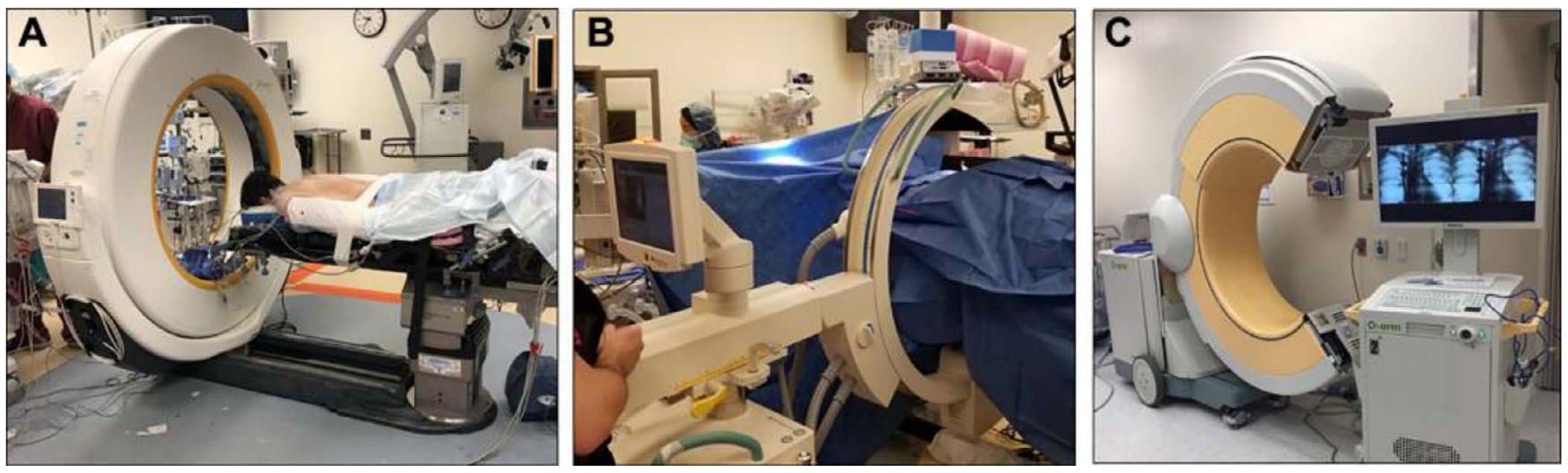

Figure 3. Technology systems for three-dimensional intraoperative computed tomography: fan beam computed tomography (FBCT) (A) and cone beam computed tomography (CBCT) (B and C). 
Table 1. Advantages and disadvantages of the different navigational systems. ${ }^{\text {a,b29 }}$

\begin{tabular}{|c|c|c|c|c|c|}
\hline & Fluoroscopy & 2D Fluoro $\mathbf{N A V}^{\mathrm{a}}$ & $3 \mathrm{D} \operatorname{cbCT}^{\mathrm{a}}$ & $3 \mathrm{D} \mathrm{iCT}^{\mathrm{a}}$ & 3D preoperative $\mathrm{CT}^{\mathrm{a}}$ \\
\hline Major advantage & $\begin{array}{l}\text { Only true real-time } \\
\text { imaging modality }\end{array}$ & $\begin{array}{l}\text { Navigation may be performed } \\
\text { using images acquired } \\
\text { intraoperatively in the } \\
\text { surgical position. Automatic } \\
\text { registration is possible. }\end{array}$ & $\begin{array}{l}\text { 3D images may be } \\
\text { acquired during } \\
\text { surgery and used } \\
\text { for navigation. }\end{array}$ & $\begin{array}{l}\text { Higher resolution } \\
\text { imaging and } \\
\text { extended scan } \\
\text { volume than } \\
\text { cbCT. }\end{array}$ & $\begin{array}{l}\text { Preoperative CT } \\
\text { may be coupled } \\
\text { to intraoperative } \\
\text { navigation system }\end{array}$ \\
\hline $\begin{array}{l}\text { Need for intraoperative } \\
\text { image acquisition? }\end{array}$ & Yes & Yes & Yes & Yes & No \\
\hline Real-time image? & Yes & Yes (if needed) & Yes (if needed) & No & No \\
\hline Screw accuracy & $\diamond \bullet$ & $\diamond$ & 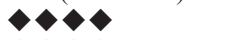 & $\diamond$ & $\diamond \bullet$ \\
\hline Bone quality image & $\diamond \diamond$ & $\diamond \bullet$ & & & \\
\hline Soft tissue quality image & & & $\diamond$ & $\diamond$ & $\diamond \triangleleft \diamond$ \\
\hline $\begin{array}{l}\text { Surgical team's radiation } \\
\text { exposure }\end{array}$ & $\diamond \diamond \diamond$ & $\diamond \diamond$ & . & . & . \\
\hline Patient's radiation exposure & $\diamond \diamond$ & $\diamond$ & $\diamond \diamond$ & $\diamond \diamond \diamond \diamond$ & 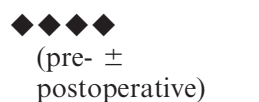 \\
\hline Cost & $\bullet$ & $\bullet$ & $\diamond \diamond$ & $\leftrightarrow$ & $\leftrightarrow$ \\
\hline $\begin{array}{l}\text { Hardware limitations (special } \\
\text { calibrated tools) }\end{array}$ & No & No & Yes & Yes & Yes \\
\hline
\end{tabular}

Abbreviations: 2D, two-dimensional; 3D, three-dimensional; cbCT, cone beam computed tomography; CT, computed tomography; fluoro NAV, fluoroscopy-guided navigation; iCT, intraoperative computed tomography.

${ }^{a}$ Table adapted with permission from ref. 29.

${ }^{\mathrm{b}} \bullet$, low; $\bullet \bullet$, medium $; \diamond \diamond \diamond$, high; $\bullet \bullet \bullet \bullet$, very high.

more demanding to handle than standard fluoroscopy systems. ${ }^{25}$ Both technologies generate excellent image quality and expose the patient to a similar amount of radiation. ${ }^{26}$ Although both modalities share many characteristics, they have a few distinct advantages and disadvantages. CBCT has to be placed and readjusted on the region of interest before each scan, making it more effortful to adjust intraoperatively. By contrast, the FBCT only needs to be centered to the gantry, which can be done by adjusting the associated OR table. Likely, the primary disadvantage of CBCT scanners is that only a few levels of the spine can be visualized during one scan without repositioning. This limits its use in procedures affecting multiple spine levels (eg, deformity surgery or multilevel fusions). The $\mathrm{CBCT}$ also has the distinct advantage of being more mobile, in that it is easier to move than an FBCT to the OR where it is needed, and no associated OR table is necessary. Additionally, CBCT scanners are cheaper for the hospital to purchase than FBCT scanners of comparable quality. Furthermore, it is possible with a CBCT to capture conventional $\mathrm{x}$-ray images in addition to the $3 \mathrm{D}$ scans acquired during the procedure, a distinct advantage over FBCT in that FBCTs are limited to 3D scans, which restricts its adaptability for different procedures. However, FBCT scanners have the advantage of visualizing more levels of the spine during the same scan and offer a superior soft tissue imaging compared with CBCT-based imaging devices. ${ }^{27}$ Another advantage of some CT scanners is the ability to use a low-dose protocol for imaging, which can also reduce the patient's radiation exposure. ${ }^{28}$ Due to its high usability and high image quality with acceptable radiation exposure, intraoperative $3 \mathrm{D} \mathrm{CT}$ imaging using FBCT or CBCT is currently the standard method in CAS. An overview of the advantages and disadvantages is shown in Table 1 .

\section{TRACKING IN NAVIGATION}

While high-quality images are essential in highquality navigation, it is only one of three essential components in high-accuracy navigation. The next and equally important part is the accuracy of the tracking of the instruments. ${ }^{15}$ This is a technically demanding task because the instruments have to be localized in a 3D space with high accuracy and at the same time be displayed in real time on the screen. To solve this task, several methods have been developed with varying degrees of popularity. ${ }^{15}$

Electromagnetic tracking was developed relatively early. In this tracking method, a field generator is placed in the immediate proximity of the patient, which induces an electromagnetic field. Via a control unit, specialized sensors within the electromagnetic field can be localized by detecting changes in the field. The sensors are attached to the patient (patient reference) as well as to the instruments. This method has the advantage of operating indepen- 

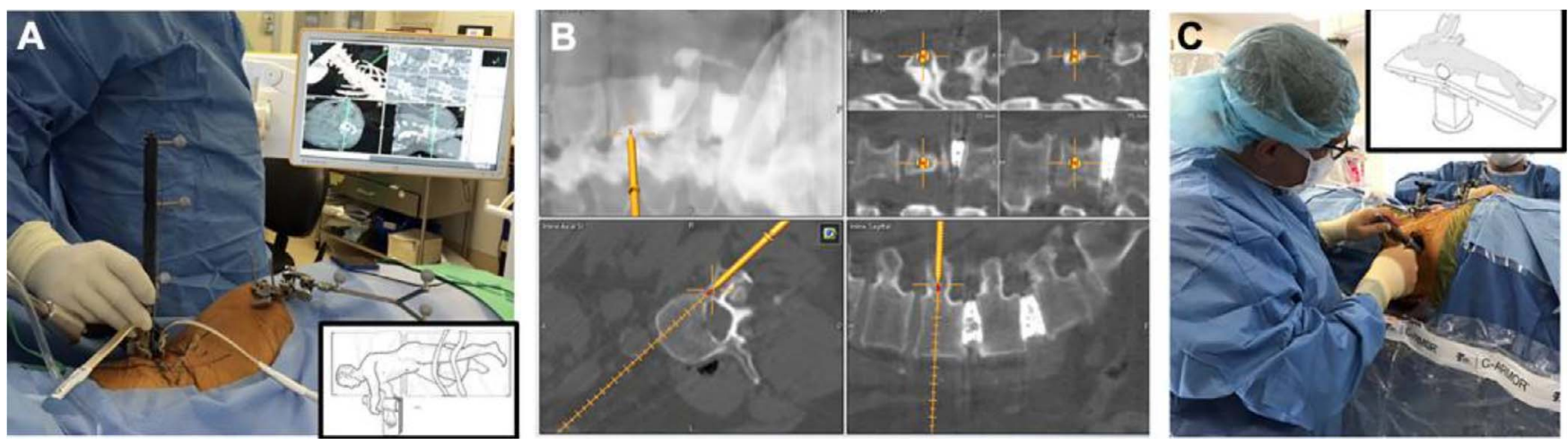

Figure 4. (A) "Total navigation" with intraoperative three-dimensional computed tomography (iCT) for single lateral position transpsoas surgery. (B) Lateral position pedicle screw placement. (C) Direct decompression in lateral position via tubular minimally invasive surgical approach.

dently of an optical connection between the navigation device and the surgical instruments. However, the key disadvantage of the method is that all ferromagnetic objects within the magnetic field interfere with the position detection of the instruments. In addition, many electromagnetic interfering factors, such as electrocautery or cell phones, can also interfere with navigation and negatively affect navigation accuracy. ${ }^{30,31}$ For this reason, magnetic systems have not been widely used in spine surgery and tend to be used in surgical specialties with a small operating field, such as cranial neurosurgery. ${ }^{30}$

Optoelectric tracking is another commonly used method for intraoperative surgical instrument tracking. In this process, orientation is provided by optical markers attached to the instruments, which are registered by a camera of the navigation unit. After calibration, the surgical instruments can be tracked in relation to the surgical field via a reference array that is attached directly to the patient and also contains optical markers., ${ }^{1,32}$ However, optoelectronic tracking does not necessarily have to be performed with visible light. Theoretically, tracking can also take place via $\mathrm{x}$ ray radiation with $\mathrm{x}$-ray-dense markers or by means of light waves from the infrared spectrum. ${ }^{5,6,32}$ The disadvantage of this technique is that this requires a direct optical connection between the camera and the reference array as well as the instrument to be tracked, which is not always easy to implement in everyday surgical practice with several people at the operating table (Figure 4). Nevertheless, optical tracking using infrared cameras has currently become one of the most common tracking methods in navigation surgery.

\section{CALIBRATION OF NAVIGATION}

In addition to high-quality images and accurate tracking of the instruments, these two elements must be accurately and precisely calibrated to facilitate navigation. To achieve the correct alignment between the patient's CT dataset and the surgical instruments in the OR, the CT dataset and the instrument position must be synchronized. ${ }^{6}$ The three most common techniques currently used are the point-matching registration technique, the surface-matching registration technique, and the automated registration technique. , $33-35^{-3}$

In the point-matching registration technique, anatomical landmarks that are easy to find intraoperatively, such as the tip of the spinous process or the tip of the transversus process, are first defined on the preoperative $\mathrm{CT}$ or magnetic resonance imaging datasets. Afterwards, these points are located with a tracking pointer in the surgical site and confirmed manually. After matching a sufficient number of anatomical points with the preoperative CT, the navigation is calibrated and can be used. This method is in general relatively fast because, in most of the cases, three points are sufficient to complete the calibration process. The disadvantage of this method is the possibility of inaccurate calibration by hurried calibration through the surgeon or soft tissue interposition, resulting in inaccurate navigation. $^{31-33}$

This procedure is therefore sometimes combined with the surface-matching registration technique. This procedure does not require preoperative determination of anatomical landmarks. The surgeon randomly selects anatomical landmarks on the bone surface and confirms them using a navigated pointer. The points are matched and aligned with the existing image dataset via the software. While 
the previously described point matching often requires only 3 points to calibrate the navigation, surface matching requires multiple points, and the accuracy increases with the number of matched points. For both methods, the points used for calibration should be as close as possible to the spinal segment that is going to be operated on to avoid inaccuracies due to movements in the vertebral joints. $^{31-33}$

The third way to calibrate the navigation is using automatic registration. With this method, an intraoperative CT scan is necessary, which is used as a reference for the navigation. During imaging, a reference array must be attached to both the CT device and the patient, which the software can use to calibrate the position of the instruments. With modern software, the calibration is performed automatically to a major extent. The surgeon only has to confirm the accuracy by positioning a tracked pointer on anatomical landmarks. ${ }^{6}$ If planning, such as screw positions, has been performed on a preoperative CT scan, the preoperative CT image data must be fused with the intraoperative CT image data. One challenge here is that preoperative imaging is usually performed in the supine position, but surgery, and thus intraoperative imaging, is performed in the prone position. ${ }^{36}$ This variability can be adjusted by modern software such as "Curvature-Correction" from Brainlab with good results in the lumbar and thoracic spine. The advantage of this calibration method is the surgeon-independent accuracy of the calibration and the short time it takes. However, the disadvantage is the higher radiation exposure, since an additional $\mathrm{CT}$ is necessary.

Another way of matching preoperative imaging to the intraoperative anatomy is the $2 \mathrm{D} / 3 \mathrm{D}$ registration technique. This method fuses a preoperative $3 \mathrm{D}$ image set (eg, a CT or magnetic resonance imaging scan) with $2 \mathrm{D}$ intraoperative fluoroscopy images. To accomplish this, a computer platform extracts geometrical features of the preoperative $3 \mathrm{D}$ dataset using image segmentation and matches the segmented image to the intraoperative 2D fluoroscopy image. The segmented dataset has a reduced amount of data, which makes the registration process relatively fast. The accuracy of this method depends immediately on the accuracy of the automated segmentation of the preoperative $3 \mathrm{D}$ dataset, which can possibly cause wrong geometrical correspondence if the image data are not segmented properly. ${ }^{37}$ Currently available robotic spine platforms (eg, the Globus ExcelsiusGPS [Globus Medical Inc, Audubon, Pennsylvania, USA]) allow the intraoperative registration with the preoperative 3D planning CT with only a lateral and an anteriorposterior image of the relevant anatomical structures.

A new approach to calibration is via machine vision image-guided surgery system, or optical topographic imaging, an automatic registration technique that does not require intraoperative CT. The technique is a combination of automatic optical calibration and classical point matching. In this system, registration is performed by two optical cameras and two infrared cameras that are integrated into the surgical lamp. During the registration process, a $2 \mathrm{D}$ pattern is projected onto the anatomy using optically visible light. The resulting optical image is registered by the optical cameras and automatically matched and calibrated in three dimensions with the preoperative dataset. This procedure is expected to be relatively fast and should increase accuracy compared with the pointmatching registration technique alone. Initial studies indicate rapid registration and comparable accuracy using this technique. ${ }^{38-40}$ However, the likelihood of successful optical registration depends on the geometry of the vertebrae and can be negatively affected from the shape of the vertebrae through which accuracy can be limited. ${ }^{41}$ This new technique offers a promising concept to reduce intraoperative radiation and provides promising data, but nevertheless further clinical studies are necessary to make a reliable statement about the actual benefit.

Another challenge for the registration technique in navigated spinal surgery is the different anatomy between preoperative imaging and actual intraoperative anatomy. Because most of the spinal surgery procedures are performed in a prone or lateral position, and the preoperative imaging is done in supine or standing positions, the spine is likely to have a different curvature on both images. While for a single vertebra, this does not cause a problem and a rigid fusion of the datasets is sufficient, for multilevel procedures, these anatomical changes need to be considered and compensated for by the fusion software. For this purpose, recent versions of navigation software offer the possibility of an elastic image fusion (eg, curvature correction Brainlab). Using this method, every vertebral body is regis- 
tered independently, and the software is able to calculate the anatomical changes between supine and prone positions and fuses the preoperative dataset with the actual position of the spine intraoperatively. ${ }^{42}$

\section{FUTURE PROSPECTS}

Considering the exponential growth in the number of publications on surgical navigation and image-guided surgery in recent years, there is some indication that this topic will become increasingly important in the future. ${ }^{43}$ Two major fields of navigated surgery that show a comparable high increase in the number of publications are augmented reality (AR) and robotics. ${ }^{6,44,45}$

AR describes a computer-generated image that can be projected onto the real-world environment. With this technology, anatomical structures can be marked on preoperative CT datasets using special software and then displayed intraoperatively. For this purpose, the preoperative planning dataset has to be fused with the intraoperative navigation CT during the surgery. After successful calibration, the preoperative marking can be projected in this way, either into the surgical microscope or into AR glasses coupled to a navigation system. There are different ways of visualizing the AR. The most common AR navigation system methods include (1) monitor-based AR, (2) microscope-based AR, (3) holographic AR, and (4) AR navigation using a head-mounted display.

AR can be visualized in various ways. Monitorbased AR describes the projection of AR onto a nearby monitor. Alternatively, the AR application can be projected within a head-mounted display; this technique (eg, Augmedics [Augmedics, Chicago, USA]) is used primarily for implanting pedicle screws. One of the notable disadvantages of this method is that a head-mounted display prevents the possibility to combine this technique with microscope-based surgical procedures. For the use of AR during procedures while using a microscope, microscope-based applications exist visualizing the AR directly in the field of view of the microscope (eg, the Brainlab implementation for Zeiss microscopes [Brainlab AG, Munich, Germany; Carl Zeiss Meditec AG, Jena, Germany]). A particular subspeciality of AR is the holographic AR, which describes a technique that projects virtual $3 \mathrm{D}$ objects to use them for AR. Holographic augmented reality has not seen extensive application in surgery, but it and the other AR navigation systems have the potential to facilitate surgical treatment techniques.

The potential applications for this technology are wide ranging. It is possible to see marked pathologies in position and size before the skin incision is made, making it easier and more precise to plan the surgical approach. Or it can be used to mark pathologies in limited vision conditions, such as those encountered in minimally invasive surgery (Figure 5, AR images). Apart from these general applications, the technology can also be used in a procedure-specific manner, marking anatomical landmarks or critical structures, such as nerves and/or blood vessels. ${ }^{6}$ In addition, AR offers a wide range of possibilities in surgical training by making it easier for trainee surgeons to orient themselves in the surgical site or even to display the next procedure step by step in the trainee surgeon's field of view, which could be particularly advantageous for more complex procedures. Overall, this technology offers a wide range of potential uses, the actual benefits of which for surgical practice will be evaluated over the next few years.

The other major IGSS-associated technology and latest development is robotic-assisted surgery. Nowadays, it is possible to plan pedicle screw position preoperatively or during the procedure using an intraoperative $\mathrm{CT}$ and to implant them with the assistance of a robotic arm. Depending on the manufacturer, the robotic support takes place in different stages. At the lowest level of robotic assistance, the surgeon must guide the robotic arm into the correct position, and the robot only makes corrections to the position to insert the screw exactly in the planned trajectory. In other variants, the robot does this more independently (Figure 6). ${ }^{6,46}$ The first studies could already demonstrate a high accuracy in robot-assisted pedicle screw implantations. ${ }^{47}$ Another study was able to show that with robotic assistance, no difference in pedicle screw placement accuracy could be detected between an experienced attending surgeon and a resident surgeon in training. ${ }^{48}$ By contrast, another study found no significant difference in the overall complication rate with the use of a surgical robot. ${ }^{49}$ The current available data do not show a consistent increase in accuracy or a better outcome using robotic assistance systems. ${ }^{48-50}$ In the past, the disadvantage of most robotic systems was that they did not allow for freehand navigation with a pointer or other instrument. Many robots offered only the 


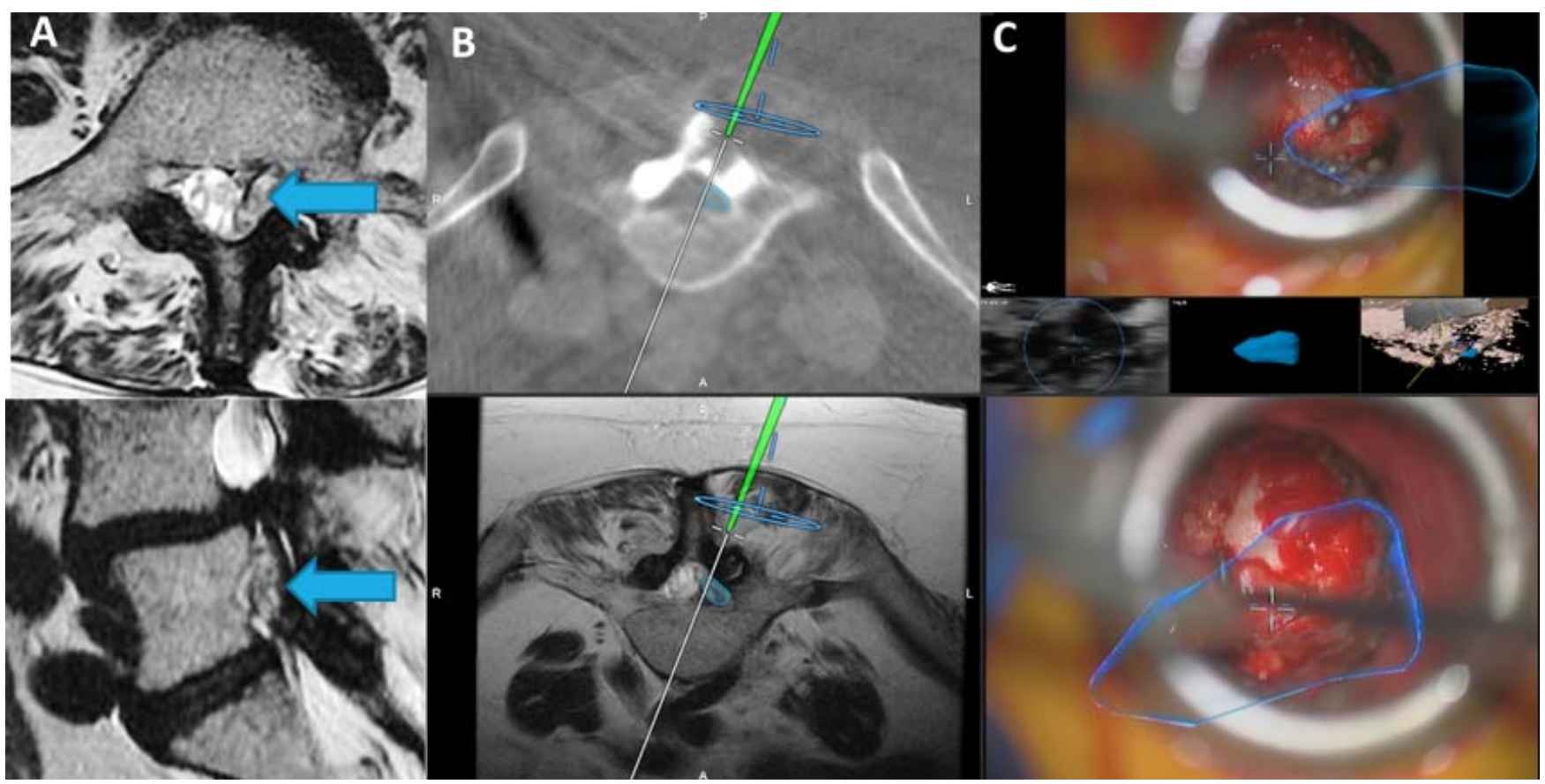

Figure 5. Example of an augmented reality-assisted spinal tumor resection in the lumbar spine. (A) Preoperative magnetic resonance imaging (MRI) with tumor (arrow). (B) Fusion of preoperative MRI and intraoperative computed tomography (iCT) with marked tumor (blue). (C) Intraoperative microscope view with tumor shape and position projected in microscope (blue).

possibility of reproducing a previously planned screw position. Because the dynamic modification of the screw trajectory to respond to the individual variations in anatomy is one of the main advantages of using freehand navigation, the most recent versions of many robotic systems, such as Mazor, Globus, and Brainlab, combine these two techniques. These image-guided surgery platforms merge the advantages of freehand navigation with the support of a robot during pedicle screw placement. Additionally, they now allow the surgeon to change planned screw sizes and trajectories intraoperatively based on variations in the patient's anatomy. The primary benefit of robotics is that it allows for planning of screw position and reproduction of said position with a high level of accuracy intraoperatively, which may reduce operative times. However, at the moment, the application of surgical robotics is limited to the placement of pedicle screws. Considering the relatively high investment costs of a surgical robot, this should not be neglected, as a technology must also be measured by its economic benefit. In addition, some currently available robot models are still very large and heavy devices, which limits handling. ${ }^{6}$ Nevertheless, many companies have dedicated their efforts to continue the development of this technology, and, in the future, further improvements of these procedures can be expected. The most recent iterations of the commonly used robotic systems (eg, Globus, Mazor [Medtronic, Dublin, Ireland], and Brainlab Cirq) offer the possibility to combine the application of
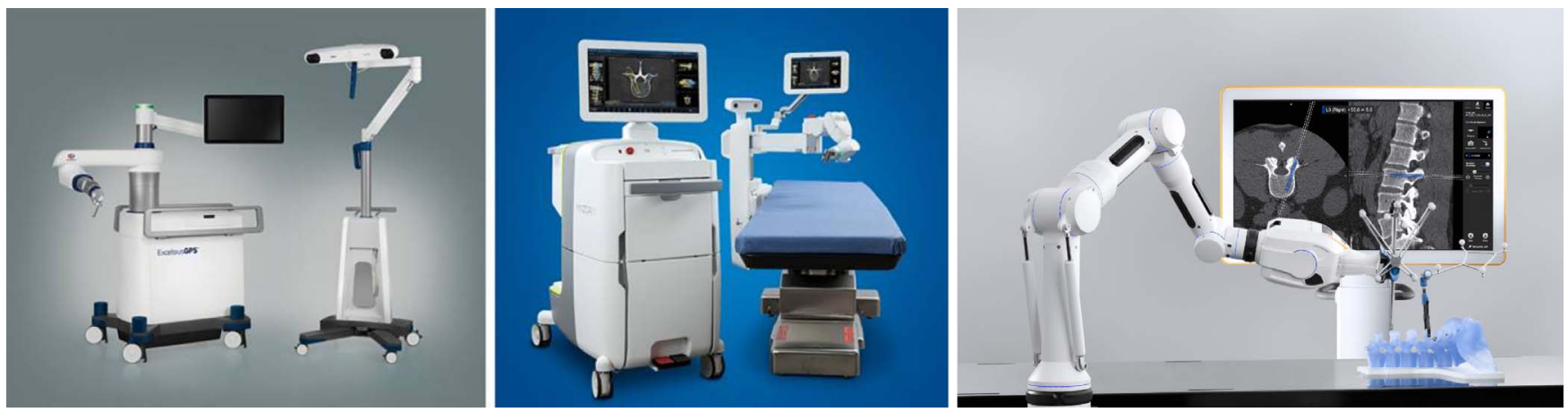

Figure 6. Examples of robots for spinal surgery, including Excelsius, Globus (left), Mazor X (middle), Cirq (Brainlab) (right). 
freehand navigation and navigated instruments in addition to the robotic-assisted procedure.$^{51-53}$ This procedure could therefore help to improve the quality of care in the future. Whether it will meet expectations remains to be seen.

\section{DISCUSSION}

The continuous improvement of IGSS has led to the procedure being widely and routinely used. ${ }^{9}$ Nevertheless, navigation also has several notable disadvantages. The use of modern $3 \mathrm{D}$ navigation requires at least preoperative sectional imaging, usually a CT. ${ }^{34}$ The most accurate calibration technique at the moment, the automatic calibration, requires an additional $\mathrm{CT}$ intraoperatively. ${ }^{6,32} \mathrm{In}$ total, this leads to a radiation exposure worth mentioning, but newer dose protocols can reduce the radiation exposure, which is why this disadvantage will become increasingly less significant. ${ }^{28}$ In addition, surgery without navigation is not free of radiation. In these cases, the orientation of the surgical level and the positioning of the implants is performed under fluoroscopy. In cases of limited visibility, such as the lower cervical or thoracic spine, multiple image shots are often required to achieve sufficient visual quality. Furthermore, it could be shown in a prospective study that in patients in whom pedicle screw implantation was performed without navigational support, a postoperative CT was performed more frequently to confirm the screw position. ${ }^{24}$ To be able to assess the actual additional radiation exposure, it is not sufficient to calculate the absolute radiation dose applied during the operation, instead the difference in the total radiation dose between conventional and navigated surgery must be taken into account. It is worth mentioning that the actual risk of developing a malignant disease from radiation exposure decreases with advancing age. ${ }^{54}$ Therefore, the risk of developing a complication due to radiation exposure must always be counterbalanced against the risk of causing a complication due to the absence of navigation. However, since misplacement of the implant can have dramatic consequences in spinal surgery, the highest possible degree of surgical accuracy should always be attempted. Additionally, there are attempts to use ultrasound for intraoperative registration with promising results, which could be a way to further decrease the amount of radiation exposure during the procedure. ${ }^{55}$
An additional drawback of IGSS is that in most of the cases, a reference array needs to be placed directly on the patient. In lumbar procedures, this is often placed with Steinman pins on the iliac crest. Even if this is done during a minimally invasive procedure, the already existing approach can be used for the pin placement, and the pins have a minimized diameter of $2 \mathrm{~mm}$, and it is still invasive. To overcome this disadvantage, companies such as Stryker (Stryker Corporation, Kalamazoo, Michigan, USA) developed reference markers that can be placed on the skin and reduce the invasiveness of the technique.

Another disadvantage of navigation is the time involved for preparation. Intraoperative calibration with the preoperative image data must be performed or an intraoperative CT must be completed. However, this one-time time investment saves further imaging by fluoroscopy later in the operation, which adds costs in time and radiation since the $\mathrm{C}$-arm has to be readjusted for each fluoroscopy shot. By contrast, a high amount of the time needed to prepare for navigation can be done in parallel with other preparation steps and therefore does not cause delays in the overall course of the surgery. In some studies, navigated procedures have resulted in shorter overall surgical times despite intraoperative $\mathrm{CT}^{56,57}$

Nevertheless, IGSS is not to be used entirely free of concerns. There is also the risk that navigation may become inaccurate due to incorrect calibration or intraoperative artifacts, such as a shifted navigation array. The smaller the deviation, the more difficult it is for the surgeon to detect, which can lead to misplacement of implants. It is therefore also important for surgeons using IGSS to have a sufficiently high level of experience to be able to detect errors and complications in the technique and to be able to react to them adequately. The surgeon, as in any technique, should never rely completely on navigation and should always critically evaluate the plausibility of the information the system provides. On principle, every surgeon using IGSS should be able to perform the same procedure without navigation, AR, or robotics. Due to technical problems, there is always a risk of failure of this technology; however, larger hospitals probably have backup equipment in most cases, reducing the probability of a total breakdown, but a small residual risk still remains. 
Another disadvantage of IGSS is that many spine surgical techniques still are not suitable for IGSS. For the lateral and anterior techniques, such as extreme lateral interbody fusion, lateral lumbar interbody fusion, oblique lumbar interbody fusion, and anterior lumbar interbody fusion, it is currently too difficult to maintain the accuracy of the navigation during the procedure due to the changes in lordosis/kyphosis as well as disc heights during cage placements. Nevertheless, a few recent studies evaluated the feasibility of robot-guided pedicle screw placement in single position lateral lumbar interbody fusion with screw placement in the lateral position and could show promising results. ${ }^{58-60}$ Seemingly, the development of new IGSS techniques is on the way to overcome these disadvantages.

An additional concern regarding the routine use of IGSS is a potentially negative impact on the training of new surgeons. As an experienced surgeon who has been trained in both navigational and conventional techniques, one might tend to use IGSS more often in some procedures because it is more convenient. This results in possibly less training of younger surgeons in surgical procedures without navigation, which should be covered by every surgeon. However, structured training, which likely exists in most hospitals, can also minimize this risk.

The cost factor of the IGSS should not be ignored. The devices must be bought, require storage space when they are not in use, and must be serviced regularly. All of these factors cost money and therefore should also be evaluated economically. Particularly for smaller hospitals with a lower number of cases in which the systems are used, this can lead to a problem justifying the purchase. A study from 2016 analyzed the costs saved due to a lower complication rate with navigation-assisted pedicle screw placement and compared these with the additional costs incurred. The result of this evaluation supports the acquisition of a navigation system also for economic reasons if the number of cases is sufficient. ${ }^{24}$

One key advantage of IGSS is the increased accuracy of the surgery. Many studies have shown that IGSS can reduce the number of screw malpositions compared with conventional surgery. ${ }^{24,61}$ In the spine, a screw malposition has serious consequences and requires correction in many cases. If this is first noticed postoperatively, it results in additional surgery. Consequently, a reduction in revision surgery rates has also been demonstrated for navigation-assisted pedicle screw implantation. $^{24}$

Like all procedures in surgery, the surgeon should try to use the procedure on the patients who are most likely to benefit from it. In addition, the surgeon should try to use IGSS in those surgical procedures where the surgeon will benefit the most. With this critical approach, the advantages of IGSS can be used efficiently while reducing the disadvantages.

\section{CONCLUSION}

IGSS has significantly evolved since its inception. One of the most notable advances has been use of fast computing to facilitate 3D navigation. Initially, allowing for better understanding of pathology via preoperative scans, this technology was translated into the OR allowing for high-quality 3D navigation intraoperatively, improving the intraoperative workflow. By improving the workflow and facilitating complex surgery, navigation is now becoming routine. IGSS can not only be used to place pedicle screws but can benefit the entire surgery. It is used for accurate planning of the skin incision through the key aspects of the surgery and even for confirmation that the goals of surgery were met through the use of a "second spin". Use of navigation throughout the surgical procedure is termed "total navigation" and has optimized the workflow, reduced the radiation exposure to surgical staff, and improved OR efficiency.

Robotic-assisted surgery provides high accuracy in the placement of pedicle screws. Robotic assistance allows for preoperative planning of the screw positions, which can potentially decrease operative time and facilitate the workload of the surgeon.

As robotics continue their own evolution with increased automation and ability to use intraoperative scans, the breadth of applications will continue to expand. Current developments point toward the future of IGSS that will be in systems that combine the advantages of "active/freehand 3D navigation" with the advantages of robotic systems.

Subsequently, AR and virtual reality technologies are enjoying new applications in supporting IGSS, including visualization of pathology or anatomically relevant structures (AR) and engaging trainees via digital anatomical 3D models (virtual reality).

Intraoperative navigation has enabled minimally invasive surgery and supported complex open 
surgeries. Although many exciting advances have already been described, we remain in the early phase of this technologic revolution. Future iterations will continue improving on current technologies while also leveraging new breakthroughs to enhance surgery and improve patient outcomes.

Finally, it is foreseeable that IGSS will find its way into all spinal surgeries and maybe also into ambulatory procedures and pain management interventions. The way for this will be to overcome the challenge of intraoperative registration and mapping of fluoroscopy and preoperative magnetic resonance imaging/CT requirements.

\section{REFERENCES}

1. Colloff E, Gleason CA, Alberts WW, Wright EW Jr. Computer-aided localization techniques for stereotaxic surgery. Confin Neurol. 1973;35(2):65-80.

2. Amirian I. The impact of sleep deprivation on surgeons' performance during night shifts. Dan Med J. 2014;61(9):1-14.

3. Chen C, Zhang X, Gu C, et al. Surgery performed at night by continuously working surgeons contributes to a higher incidence of intraoperative complications in video-assisted thoracoscopic pulmonary resection: a large monocentric retrospective study. Eur J Cardio-thoracic Surg. 2020;57(3):447-454. doi:10.1093/ejcts/ezz253

4. Bell G. Bell's law for the birth and death of computer classes: a theory of the computer's evolution. IEEE J SolidState Circuits Newsl. 2009;13(4):8-19. doi:10.1109/n-ssc.2008. 4785818

5. Weidert S, Sommer F, Suero EM, et al. Fluoroscopic marker-based guidance system improves gamma lag screw placement during nailing of intertrochanteric fractures: a randomized controlled trial. J Orthop Trauma. 2020;34(3):145150. doi:10.1097/BOT.0000000000001662

6. Hussain I, Cosar M, Kirnaz S, et al. Evolving navigation, robotics, and augmented reality in minimally invasive spine surgery. Glob Spine J. 2020;10(2 Suppl):22S-33S. doi:10.1177/ 2192568220907896

7. Hussain I, Schmidt FA, Kirnaz S, Wipplinger C, Schwartz TH, Härtl R. MIS approaches in the cervical spine. J Spine Surg. 2019;5(S1):S74-S83. doi:10.21037/jss.2019.04.21

8. Boukebir MA, Berlin CD, Navarro-Ramirez R, et al. Tenstep minimally invasive spine lumbar decompression and dural repair through tubular retractors. Oper Neurosurg. 2017;13(2):232-244. doi:10.1227/NEU.0000000000001407

9. Härtl R, Lam KS, Wang J, Korge A, Kandziora F, Audigé L. Worldwide survey on the use of navigation in spine surgery. World Neurosurg. 2013;79(1):162-172. doi:10.1016/j. wneu.2012.03.011

10. Shin BJ, James AR, Njoku IU, Härtl R. Pedicle screw navigation: a systematic review and meta-analysis of perforation risk for computer-navigated versus freehand insertion. $J$ Neurosurg Spine. 2012;17(2):111-112. doi:10.3171/2012.3. spine111098

11. Holland WW. The use of computers in surgical practice. Br J Surg. 58(10):780-783. doi:10.1002/bjs.1800581017
12. Kondziolka D, Firlik AD, Lunsford LD. Complications of stereotactic brain surgery. Neurol Clin. 16(1):35-54. doi:10 1016/s0733-8619(05)70366-2.

13. Kochanski RB, Lombardi JM, Laratta JL, Lehman RA, O'Toole JE. Image-guided navigation and robotics in spine surgery. Clin Neurosurg. 2019;84(6):1179-1189. doi:10.1093/ neuros/nyy630

14. Lavallée S, Sautot P, Troccaz J, Cinquin P, Merloz P. Computer-assisted spine surgery: a technique for accurate transpedicular screw fixation using CT data and a 3-D optical localizer. J Image Guid Surg. 1995;1(1):65-73. doi:10.1002/ (SICI)1522-712X(1995)1:1<65::AID-IGS10>3.0.CO;2-7.

15. Patel AA, Whang PG, Vaccaro AR. Overview of computer-assisted image-guided surgery of the spine. Semin Spine Surg. 2008;20(3):186-194. doi:10.1053/j.semss.2008.06. 005

16. Foley KT, Simon DA, Rampersaud YR. Virtual fluoroscopy: computer-assisted fluoroscopic navigation. Spine (Phila Pa 1976). 26(4):347-351. doi:10.1097/00007632200102150-00009

17. Holly LT, Schwender JD, Rouben DP, Foley KT Minimally invasive transforaminal lumbar interbody fusion: indications, technique, and complications. Neurosurg Focus. 2006;20(3):3-7. doi:10.3171/foc.2006.20.3.7

18. Daikh DI, Chen PP. Advances in managing ankylosing spondylitis. F1000Prime Rep. 2014;6:78(September).1-8. doi:10. 12703/P6-78

19. Brenner DJ. What we know and what we don't know about cancer risks associated with radiation doses from radiological imaging. $\mathrm{Br} J$ Radiol. 2014;87(1035):7-9. doi:10. 1259/bjr.20130629

20. Scarfe WC, Farman AG. What is cone-beam CT and how does it work? Dent Clin North Am. 2008;52(4):707-730. doi:10.1016/j.cden.2008.05.005

21. Ishikawa Y, Kanemura T, Yoshida G, et al. Intraoperative, full-rotation, three-dimensional image (O-arm)-based navigation system for cervical pedicle screw insertion: clinical article. J Neurosurg Spine. 2011;15(5):472-478. doi:10.3171/ 2011.6.SPINE10809

22. Lian X, Navarro-Ramirez R, Berlin C, et al. Total 3D Airo ${ }^{\circledR}$ navigation for minimally invasive transforaminal lumbar interbody fusion. Biomed Res Int. 2016;2016:5027340. doi:10. $1155 / 2016 / 5027340$

23. Zhang J, Weir V, Fajardo L, Lin J, Hsiung H, Ritenour ER. Dosimetric characterization of a cone-beam O-arm ${ }^{\mathrm{TM}}$ imaging system. J Xray Sci Technol. 2009;17(4):305-317. doi:10. 3233/XST-2009-0231

24. Dea N, Fisher CG, Batke J, et al. Economic evaluation comparing intraoperative cone beam $\mathrm{CT}$-based navigation and conventional fluoroscopy for the placement of spinal pedicle screws: a patient-level data cost-effectiveness analysis. Spine J. 2016;16(1):23-31. doi:10.1016/j.spinee.2015.09.062

25. King E, Daly MJ, Chan $\mathrm{H}$, et al. Intraoperative conebeam CT for head and neck surgery: feasibility of clinical implementation using a prototype mobile C-arm. Head Neck. 2013;35:959-967. doi:https://doi.org/10.1002/hed.23060

26. Lechuga L, Weidlich GA. Cone beam CT vs. fan beam CT: a comparison of image quality and dose delivered between two differing CT imaging modalities. Cureus. 2016;8(9). doi:10. 7759 /cureus. 778

27. Luís A, Navarro-Ramirez R, Kirnaz S, Nakhla J, Härtl 
R. Navigated spinal fusion. In: Phillips FM, Lieberman IH, Polly DW Jr, Wang MY, eds. Minimally Invasive Spine Surgery: Surgical Techniques and Disease Management. Cham, Switzerland: Springer International Publishing; 2019:355-374. doi:10. 1007/978-3-030-19007-1_31

28. Suero E, Strohbach O, Becker CA, et al. New mobile 3D $\mathrm{C}$-arm achieves reduced radiation exposure. Z Orthop Unfall. 2020;158(S 01):DKOU20-831. doi:10.1055/s-0040-1717775

29. Luís A, Navarro-Ramirez R, Kirnaz S, Nakhla J, Härtl R. (2019) Navigated Spinal Fusion. In: Phillips F, Lieberman I, Polly Jr. D, Wang M. (eds) Minimally Invasive Spine Surgery. Springer, Cham. https://doi.org/10.1007/978-3-030-19007-1_31

30. Suess O, Kombos T, Kurth R, et al. Intracranial imageguided neurosurgery: experience with a new electromagnetic navigation system. Acta Neurochir (Wien). 2001;143(9):927934. doi:10.1007/s007010170023

31. Hahn P, Oezdemir S, Komp M, et al. A new electromagnetic navigation system for pedicle screws placement: a human cadaver study at the lumbar spine. PLoS One. 2015;10(7):1-6. doi:10.1371/journal.pone.0133708

32. Virk S, Qureshi S. Navigation in minimally invasive spine surgery. J Spine Surg. 2019;5(S1):S25-S30. doi:10.21037/ jss.2019.04.23

33. Holly LT, Bloch O, Johnson JP. Evaluation of registration techniques for spinal image guidance. $J$ Neurosurg Spine. 2006;4(4):323-328.

34. Holly LT. Image-guided spinal surgery. Int J Med Robot Comput Assist Surg. 2006;2(1):7-15. doi:10.1002/rcs.69

35. Zheng G, Kowal J, González Ballester MA, Caversaccio M, Nolte LP. (i) Registration techniques for computer navigation. Curr Orthop. 2007;21(3):170-179. doi:10.1016/j. cuor.2007.03.002

36. Rashad A, Heiland M, Hiepe P, et al. Evaluation of a novel elastic registration algorithm for spinal imaging data: a pilot clinical study. Int $J$ Med Robot Comput Assist Surg. 2019;15(3). doi:10.1002/rcs.1991

37. Markelj P, Tomaževič D, Likar B, Pernuš F. A review of $3 \mathrm{D} / 2 \mathrm{D}$ registration methods for image-guided interventions. Med Image Anal. 2012;16(3):642-661. doi:10.1016/j.media. 2010.03.005

38. Jakubovic R, Guha D, Gupta S, et al. High speed, high density intraoperative 3D optical topographical imaging with efficient registration to MRI and CT for craniospinal surgical navigation. Sci Rep. 8(1):14894. doi:10.1038/s41598-018-32424$\mathrm{Z}$

39. Brecevich AT, Dowe C, Lebl DR, Sama AA, Abjornson C, Cammisa FP. 121. Machine-vision image guided surgery (MvIGS): an intraoperative and radiation-free spine navigation system workflow analysis. Spine J. 2019;19(9):S59. doi:10.1016/ j.spinee.2019.05.135

40. Faraji-Dana Z, Mariampillai ALD, Standish BA, Yang VXD, Leung MKK. Machine-vision image-guided surgery for spinal and cranial procedures. In: Abedin-Nasab $\mathrm{MH}$, ed. Handbook of Robotic and Image-Guided Surgery. Amsterdam, the Netherlands: Elsevier Inc; 2020:551-574. doi:10.1016/b9780-12-814245-5.00032-3

41. Guha D, Jakubovic R, Alotaibi NM, et al. Optical topographic imaging for spinal intraoperative three-dimensional navigation in mini-open approaches: a prospective cohort study of initial preclinical and clinical feasibility. World
Neurosurg. 2019;125:e863-e872. doi:10.1016/j.wneu.2019.01. 201

42. Schmidt FA, Mullally M, Lohmann M, et al. Elastic image fusion software to coregister preoperatively planned pedicle screws with intraoperative computed tomography data for image-guided spinal surgery. Int $J$ Spine Surg. 2021;15(2):295-301. doi:10.14444/8039

43. Pubmed Search Image Guided Surgery. https://pubmed. ncbi.nlm.nih.gov/?term=image+guided+surgery. Accessed February 22, 2021.

44. Pubmed Search Spine Surgery Augmented Reality. https://pubmed.ncbi.nlm.nih.gov/?term=spine+surgery+ augmented+reality. Accessed February 22, 2021.

45. Pubmed Search Spine Surgery Robotics. https://pubmed. ncbi.nlm.nih.gov/?term=spine+surgery+robotics. Accessed February 22, 2021.

46. Krieg SM, Bernhard B. First experience with the jumpstarting robotic assistance device Cirq. Neurosurg Focus. 2018;45(VideoSuppl1):V3. doi:http://thejns.org/doi/abs/10. 3171/2018.7.FocusVid.18108

47. Wallace DJ, Vardiman AB, Booher GA, et al. Navigated robotic assistance improves pedicle screw accuracy in minimally invasive surgery of the lumbosacral spine: 600 pedicle screws in a single institution. Int $J$ Med Robot. 2020;16(1):e2054. doi:10. $1002 /$ rcs. 2054

48. Vardiman AB, Wallace DJ, Booher GA, et al. Does the accuracy of pedicle screw placement differ between the attending surgeon and resident in navigated robotic-assisted minimally invasive spine surgery? J Robot Surg. 2020;14(4):567572. doi:10.1007/s11701-019-01019-9

49. Lieber AM, Kirchner GJ, Kerbel YE, Khalsa AS. Robotic-assisted pedicle screw placement fails to reduce overall postoperative complications in fusion surgery. Spine $J$. 2019;19(2):212-217. doi:10.1016/j.spinee.2018.07.004

50. Laudato PA, Pierzchala K, Schizas C. Pedicle screw insertion accuracy using O-arm, robotic guidance, or freehand technique. Spine (Phila Pa 1976). 2018;43(6):E373-E378. doi:https://doi.org/10.1097/BRS.0000000000002449

51. Lee NJ, Zuckerman SL, Buchanan IA, et al. Is there a difference between navigated and non-navigated robot cohorts in robot-assisted spine surgery? A multicenter, propensitymatched analysis of 2,800 screws and 372 patients. Spine $J$. 2021;21(9):1504-1512. doi:https://doi.org/10.1016/j.spinee. 2021.05.015.

52. Farah K, Meyer M, Prost S, et al. Robotic assistance for minimally invasive cervical pedicle instrumentation: report on feasibility and safety. World Neurosurg. 2021;150:e777-e782. doi:10.1016/j.wneu.2021.03.150

53. Farber SH, Pacult MA, Godzik J, et al. Robotics in spine surgery: a technical overview and review of key concepts. Front Surg. 2021;8(February):1-6. doi:10.3389/fsurg.2021. 578674

54. Brenner DJ, Hall EJ. Computed tomography-an increasing source of radiation exposure. $N$ Engl J Med. 2007;357(22):2277-2284.

55. Gueziri HE, Santaguida C, Collins DL. The state-of-theart in ultrasound-guided spine interventions. Med Image Anal. 2020;65:101769. doi:10.1016/j.media.2020.101769

56. Sasso RC, Garrido BJ. Computer-assisted spinal navigation versus serial radiography and operative time for 
posterior spinal fusion at L5-S1. J Spinal Disord Tech. 2007;20(2):118-122. doi:10.1097/01.bsd.0000211263.13250.b1

57. Houten JK, Nasser R, Baxi N. Clinical assessment of percutaneous lumbar pedicle screw placement using the O-arm multidimensional surgical imaging system. Neurosurgery. 2012;70(4):990-995. doi:10.1227/NEU.0b013e318237a829

58. Huntsman KT, Riggleman JR, Ahrendtsen LA, Ledonio CG. Navigated robot-guided pedicle screws placed successfully in single-position lateral lumbar interbody fusion. $J$ Robot Surg. 2020;14(4):643-647. doi:10.1007/s11701-01901034-w

59. Protopsaltis TS, Larson JJ, Frisch RF, et al. Comparison of single-position robot-assisted surgery vs conventional minimally invasive surgery following LLIF: an in vitro assessment. Spine J. 2020;20(9):S118. doi:10.1016/j.spinee.2020.05.650

60. Walker CT, Godzik J, Xu DS, Theodore N, Uribe JS, Chang SW. Minimally invasive single-position lateral interbody fusion with robotic bilateral percutaneous pedicle screw fixation: 2-dimensional operative video. Oper Neurosurg ( $\mathrm{Ha}$ gerstown, Md). 2019;16(4):E121. doi:10.1093/ons/opy240

61. Tang J, Zhu Z, Sui T, Kong D, Cao X. Position and complications of pedicle screw insertion with or without imagenavigation techniques in the thoracolumbar spine: a meta- analysis of comparative studies. J Biomed Res. 2014;28(3):228239. doi: $10.7555 /$ JBR. 28.20130159

Disclosures and COI: Roger Härtl disclosures funding from DePuy Synthes, SpineWave, Brain$\mathrm{LAB}$, and Lanx. The other authors have no conflicts and interest to declare.

Corresponding Author: Roger Härtl, MD, Department of Neurosurgery, New York Presbyterian Hospital, 525 E 68th Street, Box 99, New York, NY 10065. Phone: (212) 746-2152; Fax: (212) 7467732; Email: roh9005@med.cornell.edu.

Published 26 October 2021

This manuscript is generously published free of charge by ISASS, the International Society for the Advancement of Spine Surgery. Copyright $\odot 2021$ ISASS. To see more or order reprints or permissions, see http://ijssurgery.com. 